\title{
Self de Carreira: Estudo Longitudinal com Estudantes Universitários
}

\author{
Ana Daniela Silva \\ Maria do Céu Taveira ${ }^{1}$ \\ Eugénia Ribeiro \\ Universidade do Minho
}

\begin{abstract}
RESUMO - O self de carreira constituí um subconjunto organizado do universo cognitivo de uma pessoa, responsável pelo carácter subjetivo que a mesma confere à carreira. Este estudo pretende avaliar mudanças no conteúdo do self de carreira de estudantes universitários, do início para o final do último ano de graduação. Para tal, recorreu-se a medidas repetidas dos índices da Grelha de Repertório da Carreira (Silva \& Taveira, 2005; Silva, 2008). Na investigação, participaram 80 estudantes, dos quais 49 são mulheres $(61,25 \%)$ e 31 são homens (38,75\%), com idades entre os 21 e os 45 anos $(M=23,9, D P=4,31)$. Os resultados indicam que, no final da licenciatura, os estudantes diminuem a distância como se constrõem em relação aos outros e mantêm uma construção positiva do self de carreira.
\end{abstract}

Palavras-chave: Estudante universitário; Carreira; Self de carreira; Grelha de Repertório; Estudo Longitudinal.

\section{Career Self: A Longitudinal Study with College Students}

\begin{abstract}
The career self constitutes an organized subset of the person's cognitive universe, responsible for the subjective character that the person attributes to career. This study aims to evaluate changes in the content of the career self of college students, from the beginning to the end of last year's graduation. Therefore, we used repeated measurements of the indices of the Career Repertory Grid (Silva \& Taveira, 2005; Silva, 2008). Eighty students participated in this study, 49 women (61.25\%) and 31 men $(38.75 \%)$, aged between 21 and 45 years $(M=23.9, S D=4.31)$. The results indicate that at the end of graduation students decrease the distance they build towards others and maintain a positive construction of the career self.
\end{abstract}

Key-words: College students; Career; Career Self; Repertory Grid; Longitudinal study.

A investigação nas áreas do desenvolvimento da identidade, do auto-conceito académico e social e da auto-estima, têm demonstrado com consistência que os estudantes mudam durante a passagem pela universidade. De uma forma geral, ao longo do ensino superior, os estudantes tornam-se mais confiantes quanto às suas competências académicas e sociais e desenvolvem um maior sentido de valor pessoal e de identidade (Adams \& Fitch, 1982; Pascarella \& Terenzini, 2005; Waterman \& Waterman, 1976).

Na transição da universidade para o mercado de trabalho, estes aspectos são importantes e orientam novos ciclos de exploração e de investimento em tarefas e domínios existenciais. Referimo-nos muito em especial, às questões de identidade no âmbito da carreira, da separação da família de origem, do compromisso com relações de vinculação mais duradouras, do investimento na produtividade e da construção mais geral de um projeto de vida (Arnett, 1998, 2004, 2006, 2007; Seligman, 1994; Wortley \& Amatea, 1982). Isto é, no fundo, ao estabelecimento de si próprio como adulto.

No que concerne à construção da identidade no âmbito da carreira, as pessoas que são capazes de realizar compromissos com opções de vida, são descritas por diversos autores, como pessoas que experimentam sentimentos de continuidade entre o passado, o presente e o futuro, e estados emocionais que refletem confiança, estabilidade e otimismo em relação a esse mesmo futuro (Archer \& Waterman, 1994 conforme citado

1 Endereço para correspondência: Escola de Psicologia, Campus de Gualtar. Universidade do Minho. Braga, Portugal , 4710-057. Fone: 351-253-604671. Fax:351-253-604224.E-mail: ceuta@psi.uminho.pt em Taveira, 1997). Por seu turno, investigação suportada pelo modelo de identidade de Marcia (1966, 1980), demonstrou que o estatuto de identidade de um estudante do ensino superior não deve ser encarado como um estado permanente mas, antes, como um estado que pode sofrer alterações, em função de mudanças psicológicas internas e/ou de mudanças externas. Neste sentido, a aproximação do final de um curso de graduação pode, em si mesmo, constituir-se como um período de transição e desencadear, de fato, uma ativação da exploração, nos diferentes domínios da identidade, promovendo o estabelecimento de compromissos mais firmes no domínio vocacional, ideológico ou ético.

Por seu lado, no seio da teoria do construtivismo pessoal de Kelly (1955), a identidade ou self, define-se como um sistema de dimensões de significado, através das quais a pessoa dá sentido aos acontecimentos que antecipa e vive, no fluxo da sua existência. Este sistema desenvolve-se no contexto das relações sociais significativas e modifica-se no confronto com experiências intra e interpessoais novas. Frequentemente, no dinamismo destas interações, os indivíduos são convidados a rever o sistema de significação da sua existência, ou seja, a transformar o seu self. No entanto, para que este processo seja funcional é essencial que o sistema de significação, ou self, mantenha um sentido de continuidade e coerência (Fernandes, Senra \& Feixas, 2009). As perspectivas construtivistas mais recentes do desenvolvimento da identidade, tendem a sublinhar ainda mais o carácter transformacional do self (Brott, 2001; Lent, Brown \& Hackett, 2002). 
Este é um projeto que se atualiza no contato com os outros e com os contextos de vida, mediado, todavia, pelas limitações que as organizações cognitivas impõem à pessoa (Fernandes \& Gonçalves, 1997). De acordo com esta perspectiva, as pessoas usam as suas percepções para construir representações do mundo real e predizer e controlar os acontecimentos diários. Estas representações tomam a forma de construtos dicotomicos (competente versus incompetente). Estes, por sua vez, estão organizados em esquemas cognitivos estruturados, que permitem às pessoas interpretar os acontecimentos e desenhar o curso do seu comportamento (Kelly, 1955). As perspectivas construtivistas do desenvolvimento do self podem ser um quadro de referência útil para conceber as questões da construção do self de carreira de universitários, já que adotam uma visão relacional e socio-dinâmica do processo, congruente com a literatura vocacional mais recente (Blustein, 2008; Savickas, 2005).

Assim, é na interação com os outros que se constrói a experiência subjetiva de se sentir profissional, o self de carreira. Segundo vários autores, é de fato através das imagens que os outros devolvem de uma dada pessoa, acerca de si e do seu desempenho, ou do grau de reconhecimento e aprovação conquistados entre eles, que irá depender o maior ou menor sentido de eficácia dessa pessoa como profissional (Britzman, 1991; Bullough, Knowles \& Crow, 1991; Carrolo, 1997; Cavaco, 1995; Machado, 1996; McNally, Cope, Inglis \& Stronach, 1994; Olson \& Osborne, 1991; Simões, 1996; Simões, Santos, Gonçalves \& Ralha-Simões, 1997).

Tal como afirmam Samuel e Stephens (2000), “... o self só poderá tentar definir-se a si próprio na relação com um conjunto de outros selfs que com estes competem e interagem (p. 476)", e que não partilham, necessariamente, os mesmos princípios, valores e crenças. Assim sendo, a identidade profissional resulta de uma filtragem e aceitação de uma série de valores, comportamentos e atitudes, fundamentada nas experiências de vida do self em formação. Trata-se de um processo geralmente marcado por uma forte auto-centração, onde têm lugar a auto-análise e o auto-confronto, e no seio do qual a identidade é construída, desconstruída e reconstruída (Samuel \& Stephens, 2000). Através deste processo, o indivíduo tem a oportunidade de mudar, ou seja, renovar algumas das concepções acerca de si próprio, da profissão e dos seus objetivos de carreira. Como resultado, poderá reforçar o desejo de concretizar um projeto vocacional, ou, em alternativa, questionar-se ou agravar as suas dúvidas, ou até abandonar o seu projeto (Borralho, 2001; Machado, 1996). Portanto, esta é uma fase crucial, para estudar de que forma o mercado de trabalho em geral, e os contextos de ensino superior em particular, poderão afetar a forma como os estudantes universitários constrõem o seu self profissional e o impacto que esta construção tem no rumo que dão à sua carreira.

No âmbito da literatura vocacional, o self de carreira define o subconjunto organizado do universo cognitivo de uma pessoa que lhe permite identificar e discriminar papéis de trabalho considerados experiências fundamentais (Savickas, 2004). Esta estrutura permite às pessoas realizarem escolhas coerentes entre alternativas comportamentais. $\mathrm{O}$ self de carreira é construído com base na auto-reflexão que a linguagem torna possível e é responsável pelo caráter sub- jetivo que a pessoa confere à carreira. Ou seja, permite aos indivíduos produzirem um significado sobre a sua carreira e sobre a sua vida, e utilizarem este processo de produção de significados para orientar o seu próprio comportamento de uma forma proativa, e não apenas como uma mera reação a estímulos ambientais.

Neste contexto, o presente trabalho de investigação tem como propósito caracterizar e avaliar mudanças no conteúdo do self de carreira de estudantes do ensino superior nos anos finais de licenciatura. Para tal, recorreu-se a um estudo de medidas repetidas dos índices de uma grelha de repertório desenvolvida para o efeito - GRC (Silva \& Taveira, 2005; Silva, 2008). A grelha de repertório é uma metodologia construtivista derivada do Role Construct Repertory Test construído por Kelly (1955), com o objetivo de explorar a construção de significados pessoais, ou seja, a estrutura e o conteúdo das teorias implícitas ou redes de significado através das quais percebemos e atuamos no cotidiano. Consiste numa entrevista estruturada e orientada para a identificação das dimensões de significado que o sujeito usa para dar sentido ao seu mundo e a partir das quais se diferencia dos outros significativos (Fernandes, 2001; Winter, 2003). A informação recolhida durante a entrevista é posteriormente organizada e formalizada numa matriz que inclui as dimensões de significado identificadas (em linhas horizontais), os elementos ou outros significativos sobre os quais recai a construção de significado (em linhas verticais) e um sistema de pontuação através do qual o participante no estudo pontua cada elemento relativamente a cada significado. Dada a formalização da informação numa matriz deste tipo, esta metodologia assume o nome de grelha de repertório.

A grelha utilizada neste estudo pretende compreender o sistema de construção num âmbito específico da experiência humana - a carreira. Desta forma, a análise matemática das associações entre os elementos e construtos da grelha permite obter índices que explicam como os estudantes organizam significados acerca das suas carreiras. Perceber mudanças nesta forma de organizar a informação acerca da carreira, ao longo do último ano de licenciatura, permite-nos antecipar possíveis formas dos estudantes, mulheres e homens, lidarem com este momento significativo das suas vidas, especialmente, no que diz respeito às exigências da integração sócio-profissional que se antecipa com o finalizar de um curso. Consideramos que este é um momento de transição e por conseguinte, teoricamente, de maior ativação dos processos de exploração e compromissos envolvidos na definição do self de carreira.

\section{Método}

\section{Participantes}

A amostra é composta por 80 estudantes de graduação da Universidade do Minho, inscritos pela primeira vez no último ano do curso, no ano letivo de 2005/2006. Dos 80 estudantes, 49 são mulheres $(61,25 \%)$ e 31 são homens $(38,75 \%)$, com idades compreendidas entre os 21 e os 45 anos, sendo a média de 23,9 anos, e o desvio-padrão de 
4,31. Estes alunos provêm de 27 cursos diferentes, organizados no presente estudo, nas áreas de estudo sugeridas pela Classificação Nacional de Áreas de Educação e Formação publicadas no Diário da Republica, Portaria n²56/2005 de 16 de Março: Ciências Sociais Comércio e Direito (46\%), Educação (17\%), Artes e Humanidades (14\%), Ciências, Matemática e Informática (14\%) e, Engenharia, Indústrias Transformadoras e Construção (9\%).

A análise do nível de escolaridade dos pais e mães dos estudantes revela que uma percentagem significativa $(46,9 \%)$ possue níveis de escolaridade até o $4^{\circ}$ ano de escolaridade, sendo que os pais tendem, ligeiramente, a ter habilitações literárias superiores do que as mães dos estudantes.

\section{Instrumentos}

Grelha de Repertório da Carreira - GRC (Silva \& Taveira, 2005): consiste numa entrevista estruturada formalizada numa grelha original $16 \times 15$, em que os participantes na investigação pontuam um conjunto de elementos interpessoais elicitados pelo investigador (por exemplo, mãe, pai, irmão, professor) face a um conjunto de construtos bipolares fornecidos pelo investigador (por exemplo, Dependente dos outros vs Autónomo; Maior tendência para alcançar objetivos vs. Menor tendência par alcançar objetivos), usando uma escala de 7 pontos, sendo o valor 4 um ponto médio (por exemplo, 1 - Muito dependente dos outros e 7 - Muito Autônomo). Daqui resulta uma matriz de cotações para cada participante, com as colunas a representarem pessoas significativas do mundo interpessoal, e as linhas a representarem construtos ou dimensões de significado em torno da carreira como mostra a Figura 1. Os elementos interpessoais são elicitados e os construtos são fornecidos pelo investigador. Os 15 construtos fornecidos resultaram da revisão da literatura do desenvolvimento e escolha de carreira.

O tratamento matemático da grelha de repertório com base na análise de correspondências, permite obter uma variedade de medidas que expressam a associação entre os construtos, entre os elementos ou entre construtos e elementos.

As medidas apresentadas neste estudo são: (i) Correlação Eu.Ideal, Eu.outros e Ideal.outros; (ii) Distâncias entre o self dos estudantes e as pessoas restantes da grelha (mãe, pai, irmã/irmão; companheira/o; professor/a; pessoa significativa, homem e mulher que agrada em termos de carreira e homem e mulher que desagrada em termos de carreira); e, (iii) distâncias entre o self atual e o self em diferentes momentos no tempo: antes de entrar para a universidade, em seis meses, cinco anos e 10 anos.

A Correlação Eu-Ideal tem sido definida como uma medida de auto-estima por indicar a relação que o estudante estabelece entre o que é e o que aspira vir a ser. Uma correlação positiva entre estes dois elementos indica que os sujeitos constrõem a si próprios, em termos atuais, no mesmo sentido em que constrõem o seu Eu Ideal, o que será um bom indicador da auto-estima pessoal. Em contrapartida, correlações muito baixas ou negativas refletem uma baixa auto-estima.

A Correlação Eu-Outros, por sua vez tem sido entendida como uma medida de isolamento social auto-percebido, permitindo obter informações importantes sobre a diferenciação do self e dos Outros. O elemento Outros é um elemento construído através da média de pontuações atribuídas a todos os elementos, com exceção do Eu atual e do Eu Ideal. Valores positivos e elevados desta medida, indicam que o sujeito está a construir a sua experiência de carreira no mesmo sentido em que constrõe a dos outros elementos significativos. Em contrapartida, valores negativos e elevados indicam um elevado grau de isolamento, isto é, indicam que o sujeito se vê de forma muito diferente dos demais. Estas divergências entre o Self e os Outros têm-se observado com maior frequência em pessoas com depressão (Neimeyer \& Feixas, 1992) e em mulheres vítimas de abusos sexuais (Harter, Neimeyer \& Alexander, 1988). Em ambos os casos, este isolamento ocorre em conjugação com um índice de auto-estima baixo.

Por sua vez, os valores de correlação Ideal-Outros têm sido entendidos como uma medida de adequação percebida em relação aos outros e quanto mais elevada, neste caso, indica que os estudantes prevêem o seu ideal de carreira muito próximo e no mesmo sentido da construção que fazem da experiência de carreira dos outros significativos. Correlações negativas ou baixas refletem uma forte insatisfação com os outros que são percebidos como inadequados, ou neste caso, modelos de carreira a não seguir. Valores baixos neste índice também podem ser indicadores de um ideal muito ambicioso e pouco realista.

Com base na interpretação dos três índices apresentados, Feixas e Cornejo (1996) definem um conjunto de cinco perfis gerais de construção do self: a positividade, a superioridade, a negatividade, o isolamento e o ressentimento. A análise de perfis permite-nos compreender a forma como os sujeitos constrõem o self e tem um valor descritivo tanto mais elevado quanto mais elevadas forem as correlações positivas e negativas dos índices que o compõem. Feixas e Cornejo (1996) sugerem como ponto de corte para considerar uma correlação positiva o valor de .20 .

O perfil de Positividade caracteriza-se por uma visão globalmente positiva de si mesmo e dos outros, representada por uma correlação positiva em cada um dos três índices em análise. Quando este perfil não é acompanhado de bem-estar psicológico, pode ser um indicador de uma excessiva simplificação da realidade (e.g., considerar somente aquilo que é positivo) ou de negação dessa mesma realidade.

O perfil de Isolamento caracteriza-se por uma visão do self negativa, quando comparada com o self Ideal e com os Outros e uma relação positiva entre o Ideal e os Outros. Os sujeitos com este perfil parecem acreditar que somente eles funcionam mal em termos de carreira e gostariam de ser como os outros em termos ideais.

O perfil de Superioridade caracteriza-se por uma supremacia do self em relação aos outros. Neste caso, os sujeitos parecem construir-se muito próximos do seu ideal em termos de carreira e muito distantes dos outros em relação aos quais se pontuam num sentido oposto.

O perfil de Negatividade caracteriza os sujeitos que se definem tanto a si próprios como aos outros de forma negativa. Neste perfil, o fato do sujeito se avaliar como os outros pode reduzir o sofrimento, mas também conduzir à desesperança e pouca motivação para a mudança.

Por último, o perfil de Ressentimento, distingue-se do anterior pelo fato do sujeito se avaliar em sentido oposto 


\begin{tabular}{|c|c|c|c|c|c|c|c|c|c|c|c|c|c|c|c|c|c|}
\hline & & [вәрI ng & $ナ$ & - & - & $r$ & 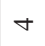 & $m$ & - & - & - & - & $\neg$ & - & - & - & $\nabla$ \\
\hline & & soue 0 I шә n日 & + & $\sim$ & - & 6 & 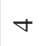 & $m$ & - & - & - & - & - & - & - & - & $n$ \\
\hline & & sour $\varsigma$ แə n马 & 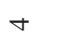 & $\sim$ & - & 6 & 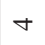 & $m$ & - & - & - & - & - & - & - & - & $n$ \\
\hline & & səsəu 9 นə n日 & 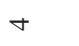 & - & - & $r$ & $\nabla$ & $m$ & - & - & - & - & - & - & - & - & $n$ \\
\hline & & 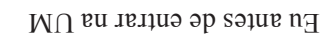 & $r$ & $\nabla$ & $\nabla$ & - & $\nabla$ & $\nabla$ & - & t & 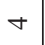 & $\nabla$ & $\nabla$ & n & $\sim$ & t & $\nabla$ \\
\hline & 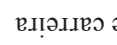 & 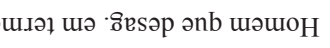 & 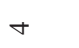 & 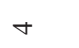 & 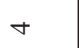 & $n$ & $\nabla$ & $\nabla$ & in & $r$ & $\sim$ & $n$ & $r$ & + & t & 6 & $\theta$ \\
\hline & в.цәц.ле & 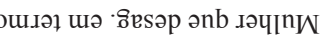 & + & t & 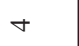 & 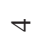 & + & 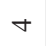 & in & $r$ & 6 & $m$ & 6 & + & + & in & $\forall$ \\
\hline & 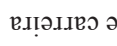 & щ.әң шә вре.ธิв әпь шәшон & $\nabla$ & $m$ & $\nabla$ & $\nabla$ & - & $\nabla$ & - & - & - & - & - & - & - & - & $\nabla$ \\
\hline & в.ı!ว.ıе & 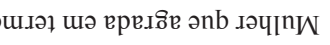 & - & - & 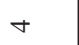 & $\nabla$ & - & $\nabla$ & - & - & - & - & - & - & - & - & $\nabla$ \\
\hline & & .ossəjo. $_{d}$ & - & - & $\checkmark$ & $\nabla$ & - & $\nabla$ & - & - & - & - & - & - & - & - & $\nabla$ \\
\hline & & oл!̣ฯчивduоoว & $\nabla$ & - & - & $\nabla$ & $\nabla$ & - & - & - & - & - & - & - & - & - & $\nabla$ \\
\hline & & 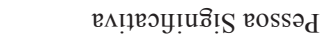 & v & - & $\sim$ & $\nabla$ & - & $\nabla$ & - & - & - & - & - & - & - & - & $\nabla$ \\
\hline & & Еृu.I/opu.II & $\nabla$ & $n$ & - & $\nabla$ & + & $\nabla$ & - & $\nabla$ & $m$ & $\nabla$ & $\nabla$ & + & $m$ & $m$ & $\nabla$ \\
\hline & & $\mathbb{E}_{\mathrm{d}}$ & $r$ & $r$ & - & $\nabla$ & $\nabla$ & $r$ & - & in & $\sim$ & $\nabla$ & $ナ$ & + & $\nabla$ & + & $n$ \\
\hline & & כQ & $r$ & $r$ & - & $\nabla$ & $\nabla$ & - & - & in & $\sim$ & $\nabla$ & $\nabla$ & $\nabla$ & $\nabla$ & $\nabla$ & - \\
\hline & & [вnџ๖ n日 & $\nabla$ & - & - & $\nabla$ & $\nabla$ & $m$ & - & - & $\sim$ & N & $\sim$ & - & - & 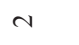 & $m$ \\
\hline $\begin{array}{l}0 \\
\Xi \\
o \\
\text { in }\end{array}$ & 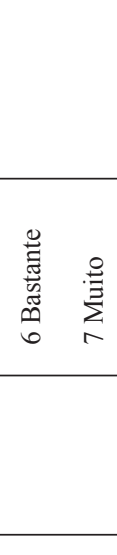 & & 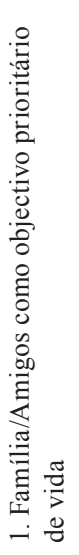 & 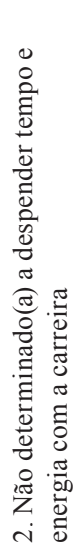 & 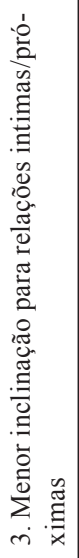 & 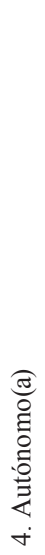 & 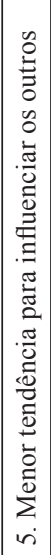 & 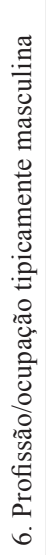 & 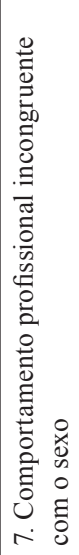 & 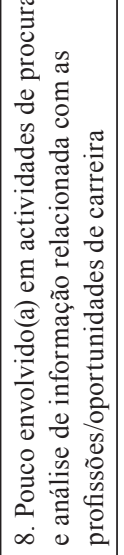 & $\begin{array}{l}0 \\
0 \\
0 \\
0 \\
0 \\
0 \\
0 \\
0 \\
0 \\
0 \\
0 \\
0 \\
\sigma\end{array}$ & 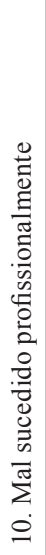 & 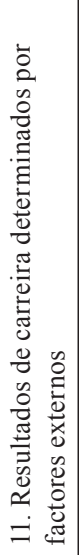 & 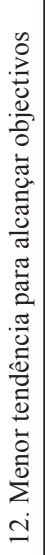 & 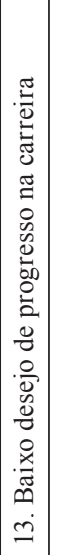 & 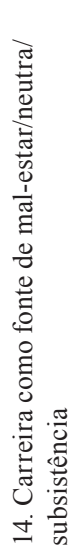 & 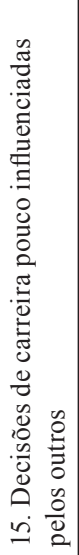 \\
\hline 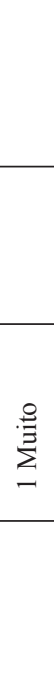 & 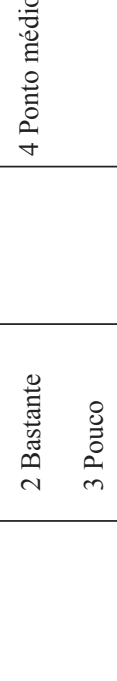 & & 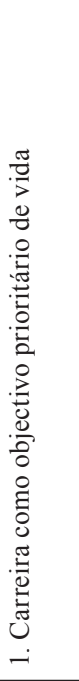 & 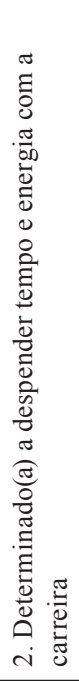 & 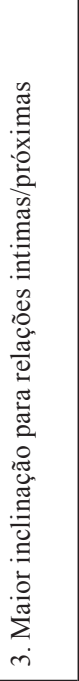 & $\begin{array}{l}0 \\
0 \\
0 \\
0 \\
0 \\
0 \\
0 \\
0 \\
0 \\
0 \\
0 \\
0 \\
00 \\
0 \\
0 \\
\dot{0}\end{array}$ & 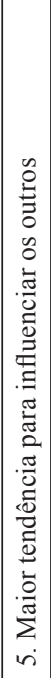 & 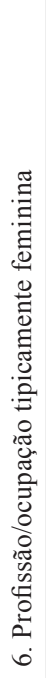 & 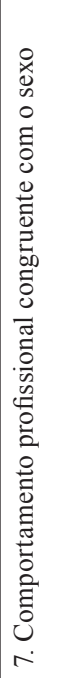 & 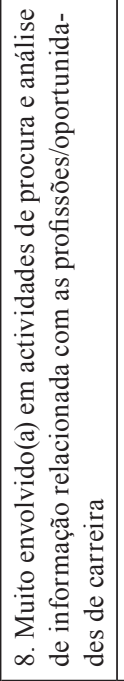 & 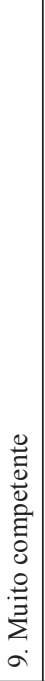 & 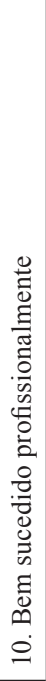 & 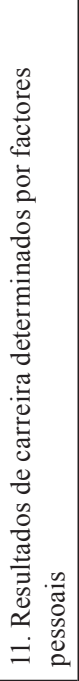 & 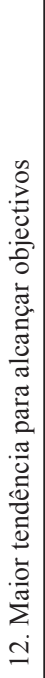 & 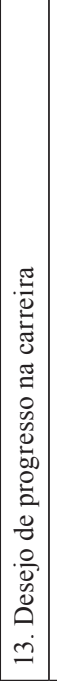 & 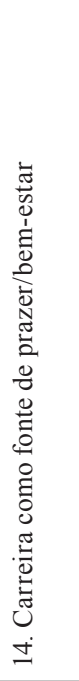 & 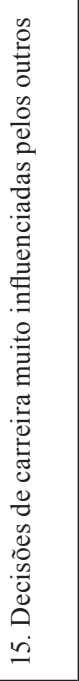 \\
\hline
\end{tabular}

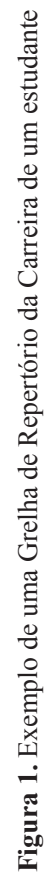


aos outros, podendo significar algum ressentimento com estes elementos.

O programa RECORD, além do cálculo de correlações entre os vários elementos ou construtos obtidos numa Grelha de Repertório, também proporciona o cálculo de distâncias entre elementos ou construtos. Estas distâncias são calculadas com base nas distâncias euclidianas e na grelha de repertório da carreira composta por 16 elementos e 15 construtos classificados numa escala de 7 pontos, as distâncias podem assumir valores entre 0 e 2,35. Quanto maior for o valor, maior é a distância entre as dimensões consideradas.

\section{Procedimento}

Os procedimentos usados para a coleta de dados obedeceram às exigências e particularidades da investigação mais ampla em que este estudo se insere (Silva, 2008), pelo que, passaremos a referir os aspectos centrais para este artigo.

A seleção da amostra resultou de duas preocupações centrais: (a) obter uma amostra equitativa nas diversas características pessoais e do contexto de vida da população a ser estudada (estudantes universitários a frequentar, pela primeira vez e sem retenções, o último ano de licenciatura); e (b) equilibrar a relação entre custos e benefícios nos procedimentos de coleta e seguimento da amostra, dada a natureza longitudinal do estudo. Por isso, foi selecionada, como instituição alvo para a realização do estudo a Universidade do Minho, dadas as facilidades antecipadas nesses procedimentos. Para a seleção dos participantes utilizou-se o método aleatório, sendo que os participantes tinham que obedecer aos seguintes critérios: (a) frequentar o último ano de uma das licenciaturas da Universidade do Minho; (b) nunca ter repetido de ano no decorrer da licenciatura; e (c) participar voluntariamente.

O plano de avaliação foi administrado em entrevistas individuais. Estas entrevistas iniciaram-se pela assinatura do termo de consentimento informado, levantamento de dados pessoais, seguindo-se a entrevista da Grelha de Repertório da Carreira (Silva \& Taveira, 2005). O tempo estimado como necessário para a entrevista individual foi de 60 minutos por cada participante. No final da entrevista, a investigadora avaliou ainda, a ativação psicológica que a administração da grelha poderia provocar nos participantes, tentando prevenir algum mal-estar, ansiedade ou desconforto decorrente dessa ativação. A avaliação ocorreu em dois momentos. $\mathrm{O}$ primeiro correspondeu ao início do ano escolar e o segundo ao final do ano escolar. Os procedimentos seguidos para a segunda fase foram os mesmos da primeira fase. Todos os participantes que concluíram as duas fases do projeto de investigação receberam um certificado de colaboração no projeto de investigação.

$\mathrm{Na}$ análise dos dados procedeu-se ao cálculo dos índices da Grelha de Repertório de Carreira com o software RECORD 4.0 (Feixas \& Cornejo, 2002) construído com base na análise de correspondências. Todas as grelhas foram tratadas individualmente neste software, tendo os resultados obtidos nos diferentes índices para cada sujeito, sido inseridos e analisados, no programa Statistic Program for Social Sciences (SPSS; versão 14.0 para Windows). No estudo de medidas repetidas, também foram utilizados os testes disponíveis no programa Statistic Program for Social Sciences (SPSS; versão 14.0 para Windows). Concretamente, depois de estudar a distribuição das variáveis em estudo, optou-se pelo T-teste de comparação de médias para amostras emparelhadas correspondentes aos dois momentos de avaliação do estudo: inicio e final do ultimo ano da licenciatura.

\section{Resultados}

A Tabela 1 apresenta os resultados do T-teste para amostras emparelhadas dos dois momentos de avaliação, para as medidas de construção do self dos estudantes: Correlação Eu-Ideal, Eu-Outros e Ideal-Outros.

Pela leitura da Tabela 1., podemos observar que os estudantes, na sua generalidade, apresentam um perfil positivo, quanto à forma como estão a construir o seu Self de carreira. Isto é, possuem uma visão globalmente positiva de si mesmos e dos outros, representada por uma correlação positiva, embora não muito elevada, em cada um dos três índices analisados. Cabe registrar também que no final da licenciatura os estudantes tendem a reduzir a correlação Eu.Ideal e Ideal. Outros e aumentam de forma estatisticamente significativa a correlação Eu.Outros. Em outras palavras, os estudantes no final da licenciatura tendem a estar menos satisfeitos com o seu Eu actual de carreira (auto-estima mais baixa), e o seu Ideal está mais longe da forma como constrõem as pessoas significativas da sua vida, contudo, sentem-se mais identificados e próximos, do ponto de vista da carreira, dessas pessoas.

Com base nas três medidas de construção do self apresentadas no quadro anterior, estudou-se a distribuição dos estudantes por diferentes perfis seguindo as indicações de Feixas e Cornejo (1996). Adicionalmente aos perfis sugeridos

Tabela 1. Comparação de medidas de Construção do Self dos estudantes nos dois momentos de avaliação (N=80)

\begin{tabular}{|c|c|c|c|c|c|c|}
\hline & \multicolumn{4}{|c|}{ Momento de avaliação } & \multirow[b]{3}{*}{$\mathrm{t}$} & \multirow[b]{3}{*}{$\mathrm{p}(\operatorname{sig})$} \\
\hline & \multicolumn{2}{|c|}{ Momento $1(\mathrm{n}=80)$} & \multicolumn{2}{|c|}{ Momento $2(n=80)$} & & \\
\hline & M & DP & M & DP & & \\
\hline R(Eu.Ideal) & 0,42 & 0,290 & 0,36 & 0,352 & 1,25 & 0,216 \\
\hline R(Eu.Outros)* & 0,47 & 0,277 & 0,54 & 0,213 & $-2,12$ & 0,037 \\
\hline R(Ideal.Outros) & 0,54 & 0238 & 0,48 & 0,271 & 1,72 & 0,089 \\
\hline
\end{tabular}

$\mathrm{p}<0,05 * * \mathrm{p}<0,01$ 
pelos autores (Positividade, Superioridade, Negatividade e Isolamento - Feixas \& Cornejo, 1996), o estudo das três medidas de construção do self nos estudantes da amostra revelou mais três perfis distintos. Estes três perfis, têm em comum o fato de sugerirem construções conflituosas das dimensões do Self, uma vez que, a satisfação de uma das correlações colide com a satisfação de outras. Os perfis apresentados, espelham as relações de conflito referidas por Slade e Sheehan (1979) a respeito das relações entre construtos, em particular, quando o autor se refere à incompatibilidade numa tríade com duas relações positivas e uma negativa. Por exemplo, o primeiro perfil a que chamamos Aproximação Social, caracteriza-se por uma correlação negativa Eu.Ideal e, positiva nas medidas Eu.Outros e Ideal.Outros, o que sugere que os estudantes estão insatisfeitos com o seu Self de carreira mas constrõem o seu self actual de carreira no mesmo sentido da forma como constrõem as outras pessoas.

Com efeito, o estudante parece construir-se na direção de algo que vê no mesmo sentido do seu ideal, mas não se vê no mesmo sentido desse ideal, o que parece refletir uma construção conflituosa das dimensões do self. O segundo perfil, que denominamos de Aspiração de Diferenciação, caracteriza-se por uma correlação positiva Eu.Ideal. e Eu. Outros e, uma correlação negativa Ideal.Outros. Este perfil sugere que os estudantes apesar de se construírem no sentido daquilo que desejam em termos ideais e no mesmo sentido da forma como constrõem as outras pessoas, revelam um desejo de em termos ideais, serem diferentes das outras pessoas, diferenciando-se destas em termos de carreira. Também este perfil revela conflito na construção das dimensões do self, uma vez que, o estudante constrói-se no mesmo sentido que os outros e que o seu ideal, mas são estas as dimensões que ele constrói em sentidos opostos. Por último, o terceiro perfil identificado, foi denominado de Confusão por se caracterizar por uma relação aparentemente incongruente entre as três medidas, demonstrando que os estudantes não são capazes no momento de definir o seu self de forma consistente parecendo querer que o seu ideal vá de encontro a algo que de momento eles vêem no sentido oposto ao seu self que, por sua vez, está em sintonia com o que desejam em termos ideais. Caracteriza-se por uma relação positiva entre Eu.Ideal, negativa entre o Eu.Outros e positiva entre o Ideal. Outros.

A Tabela 2 apresenta a distribuição dos estudantes pelos diferentes perfis de construção do self referidos.

Como se pode observar pela leitura da Tabela 2, no momento 1 e 2, os estudantes, distribuem-se em maioria pelo perfil de Positividade. O segundo perfil com frequências mais elevadas, diz respeito a um dos perfis não definidos, previamente, na literatura, o Perfil de Aproximação Social. Vale registrar ainda, que no final da licenciatura, ao contrário do que se verifica no inicio do ano letivo, não se registra nenhum estudante, mulher ou homem, com o perfil de Superioridade, o que pode sugerir que o final da licenciatura aproxima o Self de carreira dos estudantes do das outras pessoas.

A Tabela 3 por sua vez, apresenta os resultados do teste de medidas emparelhadas entre os dois momentos de avaliação do estudo, para as medidas de distância entre o Self dos estudantes e outras pessoas significativas.

No que diz respeito às distâncias entre o self dos estudantes e outras pessoas significativas do ponto de vista da
Tabela 2. Distribuição dos estudantes pelos Perfis de Construção do self, nos dois momentos de avaliação $(\mathrm{N}=80)$

\begin{tabular}{lcc}
\hline & Momento 1 n(\%) & Momento 2 n(\%) \\
\hline Positividade & $51(63,8)$ & $47(58,8)$ \\
\hline Superioridade & $4(5,0)$ & $0(0,0)$ \\
\hline Negatividade & $4(5,0)$ & $7(8,8)$ \\
\hline Isolamento & $6(7,5)$ & $4(5,0)$ \\
\hline Aproximação Social & $10(12,5)$ & $16(20,0)$ \\
\hline Aspiração Diferenciação & $2(2,5)$ & $4(5,0)$ \\
\hline Confusão & $3(3,8)$ & $2(4,1)$ \\
\hline
\end{tabular}

carreira, na Tabela 3, pode-se verificar que no momento 1 , os elementos que os estudantes constrõem como mais distantes do seu Self de carreira são, a mulher que desagrada em termos de carreira, o homem que desagrada em termos de carreira e a mãe, respectivamente. Em contrapartida, as pessoas que os estudantes constrõem como mais próximas do self são o/a companheiro/a e a mulher que agrada do ponto de vista da carreira. Vale ressaltar que as modelos mulheres parecem ser vistas, em termos positivos, como os mais próximos ou então, quando em desagrado, como as mais distantes do self dos estudantes. Comparando os dois momentos de avaliação do estudo, verifica-se que os estudantes, diminuem as distâncias relativamente a todos as pessoas, e fazem-no de forma estatisticamente significativa no caso da mãe, pai, pessoa significativa, professor/a, mulher que desagrada e homem que desagrada. Tal dado reforça a ideia, já referida noutros índices, de que no final da licenciatura os estudantes se constrõem mais próximos dos outros do que no início do ano letivo. Com efeito, o final da licenciatura parece introduzir mudanças na forma como os estudantes percebem os outros em relação a si mesmos, em especial no que diz respeito aos pais, professores e modelos negativos de carreira. As relações íntimas (irmão, companheiros) e os modelos positivos (mulher e homem que agrada) parecem não sofrer mudanças tão acentuadas.

A Tabela 4 apresenta os resultados para as medidas de distância entre elementos temporais do Self dos estudantes.

Pela leitura da Tabela 4 podemos constatar que nos dois momentos de avaliação, os estudantes constrõem-se, em termos de perspectiva temporal, como mais distantes daquilo que foram no passado (antes da entrada na universidade), e mais próximos daquilo que serão no futuro, a curto prazo (seis meses), médio prazo (cinco anos) e longo prazo (10 anos), respectivamente. Tal, indica que os estudantes possuem uma perspectiva de alguma mudança gradual em termos de carreira ao longo dos anos. Contudo, se considerarmos que numa grelha $15 \times 16$, com uma escala de 7 pontos, o valor de distância varia entre 0 e 2,35, podemos considerar que em qualquer das medidas, os estudantes da amostra registram valores baixos de distância. Este aspecto, sugere congruência/ continuidade na construção do self de carreira dos estudantes ao longo do tempo. É importante destacar ainda, que apesar das diferenças de médias das distâncias, entre os dois momentos, não serem estatisticamente significativas, verifica-se uma tendência para a redução de todas as médias no segundo momento de avaliação do estudo. 
Tabela 3. Comparação de medidas de distância entre o Self dos estudantes e outras pessoas significativas nos dois momentos de avaliação (N=80)

\begin{tabular}{|c|c|c|c|c|c|c|}
\hline & \multicolumn{4}{|c|}{ Momento de avaliação } & \multirow[b]{3}{*}{$\mathrm{t}$} & \multirow[b]{3}{*}{$\mathrm{p}(\mathrm{sig})$} \\
\hline & \multicolumn{2}{|c|}{ Momento $1(\mathrm{n}=80)$} & \multicolumn{2}{|c|}{ Momento $2(\mathrm{n}=80)$} & & \\
\hline & M & DP & M & DP & & \\
\hline Eu-mãe** & 0,51 & 0,279 & 0,45 & 0,262 & 2,74 & 0,008 \\
\hline Eu-Pai* & 0,38 & 0,245 & 0,33 & 0,201 & 2,45 & 0,017 \\
\hline Eu-irmã/irmão & 0,26 & 0,197 & 0,23 & 0,195 & 1,25 & 0,212 \\
\hline Eu-pessoa significativa* & 0,29 & 0,230 & 0,23 & 0,202 & 2,56 & 0,012 \\
\hline Eu-Companheiro/a & 0,23 & 0,193 & 0,19 & 0,151 & 1,92 & 0,058 \\
\hline Eu-Professor/a** & 0,26 & 0,186 & 0,20 & 0,143 & 2,81 & 0,006 \\
\hline Eu-Mulher que agrada & 0,23 & 0,168 & 0,20 & 0,140 & 1,76 & 0,083 \\
\hline Eu-Homem que agrada & 0,25 & 0,178 & 0,22 & 0,147 & 1,55 & 0,126 \\
\hline Eu-Mulher que desagrada* & 0,62 & 0,370 & 0,51 & 0,346 & 2,64 & 0,010 \\
\hline Eu-Homem que desagrada* & 0,59 & 0,303 & 0,50 & 0,309 & 2,55 & 0,013 \\
\hline
\end{tabular}

Tabela 4. Comparação de medidas de distância entre elementos temporais do self dos estudantes nos dois momentos de avaliação (N=80)

\begin{tabular}{|c|c|c|c|c|c|c|}
\hline & \multicolumn{4}{|c|}{ Momento de avaliação } & \multirow[b]{3}{*}{$\mathrm{t}$} & \multirow[b]{3}{*}{$\mathrm{p}(\mathrm{sig})$} \\
\hline & \multicolumn{2}{|c|}{ Momento $1(\mathrm{n}=80)$} & \multicolumn{2}{|c|}{ Momento $2(n=80)$} & & \\
\hline & M & DP & M & DP & & \\
\hline Eu.Eu antes de entrar na Universidade & 0,25 & 0,207 & 0,23 & 0,215 & 1,03 & 0,307 \\
\hline Eu.Eu em 6 meses & 0,12 & 0,151 & 0,09 & 0,117 & 1,23 & 0,222 \\
\hline Eu. Eu em 5 anos & 0,15 & 0,149 & 0,11 & 0,117 & 1,78 & 0,079 \\
\hline Eu.Eu em 10 anos & 0,18 & 0,144 & 0,15 & 0,135 & 1,61 & 0,112 \\
\hline
\end{tabular}

$* \mathrm{p}<0,05 * * \mathrm{p}<0,01$

\section{Discussão}

No que diz respeito às medidas de conteúdo dos estudantes, salienta-se o perfil de positividade que parece caracterizar a construção do self da maioria dos estudantes. Este aspecto parece ir de encontro aos resultados obtidos na investigação realizada junto a estudantes universitários, com base no modelo de identidade de Marcia (1966, 1980), que sugerem a mudança da identidade, ao longo dos anos de frequência do ensino superior, no sentido de uma resolução mais positiva das questões de desenvolvimento psicossocial (Adams \& Fitch, 1982; Waterman \& Waterman, 1976). Contudo, cabe salientar, a este respeito, a identificação, nestes estudantes, de três novos perfis de construção do self, que espelham conflito. Sobressai aqui, em especial, o perfil de Aproximação Social, que chega mesmo a ser o segundo mais verificado na amostra de estudantes. Com efeito, os estudantes parecem ir buscar algum conforto na identificação com os outros que, possivelmente também enfrentam dificuldades semelhantes, embora isso implique uma perda de auto-estima.

Nesta fase do desenvolvimento dos estudantes do ensino superior, a construção de si mesmos e dos outros em termos de carreira, assume particular relevância. Possuir um trabalho valorizado social e pessoalmente, ou a percepção dessa possibilidade, aumenta a auto-estima, ajudando no desenvolvimento de um sentido de identidade seguro e estável, que pode refletir num perfil de positividade em termos de construção do self. No entanto, o trabalho ou o insucesso na obtenção de um emprego, pode também ser fonte de frustração e de stress, contribuindo negativamente para o auto-conceito e para a realização pessoal dos indivíduos, assim como, para a criação de stress adicional noutras áreas de vida (Moos, 1986 conforme citado por Conger, 1991). O sentimento de que não se é necessário e de que, o que se quer não está disponível, promove a dúvida, ressentimentos e uma diminuição de auto-estima, aumentando a probabilidade do desenvolvimento de uma identidade confusa ou até negativa (Conger, 1991). Esta identidade, em termos da construção do self pode se refletir, por exemplo, em perfis de negatividade ou nos perfis de conflito identificados.

Um último aspecto importante a ser destacado em relação aos perfis de construção de self, prende-se com o fato de, ao contrário do que se verificou no inicio do ano letivo, no final da licenciatura, nenhum estudante pontuou no perfil de Superioridade. Com efeito, o contato com o mercado de trabalho, parece ajustar a construção do self dos estudantes à forma como vêem os outros em termos de carreira. A passagem do papel de estudantes para trabalhadores, pode implicar também a perda de algumas dimensões de carreira desejáveis, mas que a generalidade das outras pessoas também não possui. Em outras palavras, estes resultados parecem sugerir que no final da licenciatura as construções dos estudantes se revestem de maior realismo. 
Estes aspectos de aproximação/distância aos outros significativos, nos dois momentos de estudo, pode ainda ser observado nos resultados relativos às distâncias entre 0 self dos estudantes e outras pessoas significativas, também contempladas neste estudo. Com efeito, verifica-se que no final da licenciatura os estudantes, diminuíram as distâncias relativamente a todas as pessoas. A este respeito, Harter (1999, 2003) refere o self como uma construção cognitiva e social, uma teoria que o indivíduo constrói sobre si próprio que se vai desenvolvendo ao longo do tempo, em função de evoluções cognitivas e de elementos sociais como os valores, a retroação social e a fonte de comparação proporcionada pelos outros. Logo, compreender a forma como os estudantes se constrõem em interação com outros significativos reveste-se de especial importância. Neste contexto, sobressai o fato dos pais dos estudantes, especialmente as mães, serem uma das pessoas da grelha que apresentam maiores distâncias em relação à forma como os estudantes constrõem o seu self. Estes dados, podem ser explicados se levarmos em atenção os dados demográficos da amostra em estudo.

Efetivamente, a maioria dos pais da amostra possuem níveis educacionais e estatutos profissionais baixos, o que pode explicar a distância em termos de construção do self de carreira por estes estudantes. Alias, esta é uma tendência demográfica em termos da população estudantil, essencialmente a partir da década de 60 até a atualidade, em que verificamos que, embora entre as décadas de 60 e 90, se tenha registrado um aumento das taxas de escolarização da população portuguesa em geral, se registrou também o alargamento da base social de recrutamento dos jovens do ensino superior, com uma incidência crescente das famílias com baixos níveis de capital sócio-cultural (Balsa, 1997; Balsa, Simões, Nunes, Carmo \& Campos, 2001; Braga da Cruz e cols., 1995; Fernandes, 2001). Assim, ao contrário dos seus pais, estes estudantes atingiram o ensino superior e aspiram estatutos profissionais mais elevados. Os estudantes parecem utilizar os pais como modelos negativos (Gibson, 2004), ou seja, o esforço e dificuldades que os pais enfrentam podem os ter levado a fazer outras opções e construírem o seu self de carreira de forma distinta. Apesar deste afastamento em termos de identificação, provavelmente, o suporte e encorajamento parental para o desenvolvimento de carreira dos filhos, neste caso, pode ter sido um fator moderador que explica o sucesso destes estudantes.

Outro dado importante é que no final da licenciatura, se obteve uma redução da distância estatisticamente significativa no caso da mãe, pai, pessoa significativa, professor/a, mulher que desagrada e homem que desagrada. Este fato pode revelar que a entrada no mercado de trabalho aumenta a identificação dos estudantes com as figuras parentais e modelos negativos. Além disso, o aumento da proximidade à figura do professor revela a importância destas figuras no desenvolvimento de carreira dos estudantes, para além da permanência nas instituições de ensino. De registrar ainda, que os modelos mulheres parecem ser vistos, em termos positivos, como os mais próximos, ou então, quando em desagrado, os mais distantes do self dos estudantes. Este aspecto é curioso, uma vez que no processo de elicitação dos elementos da grelha, a mulher que agrada e a mulher que desagrada em termos de carreira, eram aqueles que os estudantes demonstravam mais dificuldade em indicar, contudo são também aqueles que os estudantes mais diferenciam em termos do que identificam ou não identificam nos seus selves. Desta forma, a falta de modelos femininos referida na literatura (McLure \& Piel, 1978; O’Donnell \& Anderson, 1978; O’Leary, 1974), pode por um lado limitar os processos de modelagem das mulheres em termos profissionais, e por outro, também, tornar mais salientes os poucos modelos que existem, favorecendo o processo de identificação, de mulheres e homens, com esses modelos.

Outro aspecto saliente neste conjunto de resultados sobre as distâncias prende-se com a proximidade com que os estudantes, se constrõem em termos de carreira em relação aos/às Companheiros/as, justificando o impacto que estas relações intimas podem ter no desenvolvimento de carreira de mulheres e homens, bem como a importância da dimensão de intimidade e da conjugação da mesma em termos de carreira, no desenvolvimento do jovem adulto.

Se considerarmos a noção recente de modelo social, como construções cognitivas ativas desenvolvidas pelos indivíduos para construir os seus selves ideais ou possíveis baseados nas suas necessidades e objetivos de desenvolvimento (Bucher \& Stelling, 1977; Cross \& Markus, 1991; Ibarra, 1999; Markus \& Nurius, 1986), podemos afirmar que os resultados das distâncias obtidos com a GRC concorrem para a compreensão de como estes modelos vão atuando de forma diferencial à medida que os jovens estudantes do ensino superior vão progredindo em termos do seu desenvolvimento de carreira.

Por último, analisando as distâncias que os estudantes percebem em relação aos seus selves, em diferentes momentos no tempo verifica-se que os estudantes possuem uma perspectiva de alguma mudança gradual em termos de carreira ao longo dos anos. Segundo Harter (1999, 2003), o jovem preocupa-se com os selves futuros, exprimindo nas suas escolhas - de carreira, por exemplo - valores e crenças próprios, sugerindo-se aqui um paralelismo com o que Markus e Nurius (1986, p. 954) designaram selves possíveis, enquanto derivados das representações do indivíduo no passado mas incluindo representações de si no futuro. Com efeito, os jovens da amostra parecem incluir esta perspectiva temporal na forma como constrõem o seu self, revelando uma tendência, entre os dois momentos de avaliação do estudo, para a redução das médias de distância entre os elementos do self em diferentes momentos no tempo. Tal sugere que, no final da licenciatura, os estudantes percebem-se mais semelhantes do ponto de vista da carreira, em relação ao que eram antes de entrar na universidade, bem como, percebem menos mudanças ao longo do tempo em termos do seu self de carreira, o que poderá indicar que nesta fase se encontram mais comprometidos do ponto de vista da identidade vocacional, e menos disponíveis para mudar. Este aspecto, aliás, vai de encontro às ideias de Marcia $(1966,1980)$, que defende que o período correspondente à frequência do ensino superior é, por excelência, um período que leva os jovens a explorar de forma mais ampla e variada as questões associadas à construção e definição da sua identidade e a assumir compromissos mais firmes e definitivos nas áreas vocacional, ideológica e interpessoal/sexual das suas vidas.

Concluindo, o presente estudo permitiu verificar que ao longo do último ano de frequência do ensino superior os estu- 
dantes tendem a desenvolver um perfil positivo de construção do self de carreira e constrõem-se de forma mais semelhante às pessoas significativas da sua vida o que lhes permite uma maior identificação social. Consideramos que perceber estes aspectos do conteúdo da construção do self dos estudantes revela-se útil para, por exemplo, melhor planejar intervenções de gestão pessoal de carreira com esta população.

\section{Referências}

Adams, G., \& Fitch, S. (1982). Psychological environments of university departures: Effects on college students identity status and ego stage development. Journal of Personality and Social Psychology, 44, 1266-1275.

Arnett, J. J. (1998). Learning to stand alone: The contemporary American transition to adulthood in cultural and historical context. Human Development, 41, 295-315.

Arnett, J. J. (2004). Emerging adulthood: The winding road from the late teens through the twenties. New York: Oxford University Press.

Arnett, J. J. (2006). Emerging adulthood: Understanding the new way of coming of age. In J. J. Arnett \& L. L. Tanner (Eds.), Emerging adults in America: Coming of age in the $21^{\text {st }}$ century (pp.3-19). Washington, DC: APA Books.

Arnett, J. J. (2007). Suffering, selfish, slackers? Myth and reality on emerging adults. Journal of Youth and Adolescence, 36, 23-29.

Balsa, C. (Ed.) (1997). O perfil sócio-económico dos estudantes do Ensino Superior. Lisboa: Universidade Nova de Lisboa, CNACES/CEOS.

Balsa, C., Simões, J. A., Nunes, P., Carmo, R., \& Campos R. L. (2001). Perfil dos estudantes do Ensino Superior: Desigualdades e diferenciação. Lisboa: CEOS, Edições Colibri.

Blustein, D. L. (2008). The role of work in psychological health and well-being: A conceptual, historical, and public policy perspective. American Psychologist, 63, 228-240.

Borralho, A. M. A. (2001). Didáctica da Matemática e formação inicial: Um estudo com três futuros professores. Tese de Doutorado, Universidade de Évora, Évora.

Braga da Cruz, M., Cruzeiro, M. E. Ramos, A., Leandro, E., Nunes, J. S., Matias, N., Pedroso, P., Robinson, M. G., \& Cavaco, V. (1995). O desenvolvimento do Ensino Superior em Portugal: Situação e problemas de acesso. Lisboa: Departamento de Programação e Gestão Financeira, Ministério da Educação.

Britzman, D. P. (1991). Practice makes practice: A critical study of learning to teach. Albany: State University of New York Press.

Brott, P.E. (2001). The storied approach: A postmodern perspective for career counseling. Career Development Quarterly, 49, 304-313.

Bucher, R., \& Stelling, J. G. (1977). Becoming professional. Beverly Hills, CA: Sage.

Bullough, R. V., Jr., Knowles, J. G., \& Crow, N. A. (1991). Emerging as a teacher. London: Routledge.

Carrolo, C. (1997). Formação e identidade profissional dos professores. In M. T. Estrela (Ed..), Viver e construir a profissão docente. Porto: Porto Editora, Colecção Ciências da Educação.

Cavaco, M. H. (1995). Ofício do professor: O tempo e as mudanças. In A. Nóvoa (Ed.), Profissão professor (2 $2^{\mathrm{a}}$ ed., pp.155-191). Porto: Porto Editora.
Conger, J. J. (1991). Adolescence and youth: Psychological development in a changing world ( $4^{\text {th }}$ ed.). London: Harper Collins Publishers.

Cross, S., \& Markus, H. (1991). Possible selves across the life span. Human Development, 34, 230-255.

Decreto-Lei Portaria no 256 (2005, 16 de Março). Define a Classificação Nacional de Áreas de Educação e Formação. Lisboa: Diário da República Portuguesa.

Feixas, G., \& Cornejo, J. M. (1996). Manual de la técnica de Rejilla mediante el programa RECORD v. 2.0. ( $2^{\mathrm{a}} \mathrm{ed}$, revisada y ampliada). Barcelona: Paidós.

Feixas, G., \& Cornejo, J. M. (2002). RECORD v. 4.0: Análisis de correspondencias de constructos personales. Reitrado em 27/05/2010, de http://www.terapiacognitiva.

net/record.

Fernandes, E. (2001). Grelha de Repertório. In E. Fernandes \& L. Almeida (Eds.), Métodos e Técnicas de Avaliação: Contributos para a prática e investigação psicológicas (pp. 77-108). Universidade do Minho, Centro de estudos em Educação e Psicologia.

Fernandes, E., \& Gonçalves, O. (1997). Exploração de construtos pessoais sobre o self em mudança: um estudo preliminar com sujeitos depressivos e agorafóbicos. Psicologia: Teoria, Investigação e Prática, 2, 33-52.

Fernandes, E., Senra, J., \& Feixas, G. (2009). Psicoterapia Construtivista: Um modelo centrado em dilemas. Col. Cadernos de Psicoterapia. Braga: Psiquilíbrios Edições.

Gibson, D. (2004). Role models in career development: New directions for theory and research. Journal of Vocational Behavior, 65, 134-156.

Harter, S. (1999). The construction of the self: Developmental perspective. New York: The Guilford Press.

Harter, S. (2003). The development of self representations during childhood and adolescence. In M. Leary \& J. Tangney (Eds.), Handbook of self and identity (pp. 610-642). New York: The Guilford Press.

Harter, S., Neimeyer, R. A., \& Alexander, P. (1988). Long-term effects of incestuous child abuse in college women: social adjustment, social cognition, and family characteristics. Journal of Consulting and Clinical Psychology, 56, 5-8.

Ibarra, H. (1999). Provisional selves: Experimenting with image and identity in professional adaptation. Administrative Science Quarterly, 44, 764-791.

Kelly, G. A. (1955). The psychology of personal constructs (vols. I, II). New York: W. W. Norton.

Lent, R. W., Brown, S. D., \& Hackett, G. (2002). Social cognitive career theory. In D. Brown \& Associates (Eds.), Career choice and development (4th ed., pp. 255-311). San Francisco: Jossey-Bass.

Machado, C. G. (1996). Tornar-se professor - Da idealização à realidade. Tese de Doutorado, Universidade de Évora, Évora.

Marcia, J. E. (1966). Development and validation of ego-identity status. Journal of Personality and Social Psychology, 3, 551559.

Marcia, J. E. (1980). Identity in adolescence. In J. Adelson (Ed.), Handbook of adolescence psychology (pp.159-187). New York: Wiley.

Markus, H., \& Nurius, P. (1986). Possible selves. American Psychologist, 41, 954-969. 
McLure, G., \& Piel, E. (1978). Career-bound girls and science careers: Perceptions of barriers and facilitating factors. Journal of Vocational Behavior, 12, 172-183.

McNally, J., Cope, P., Inglis, B., \& Stronach, I. (1994). Current realities in the student teaching experience: A preliminary inquiry. Teaching and Teacher Education, 10(2), 219-231.

Neimeyer, R. A., \& Feixas, G. (1992). Cognitive assessment in depression: A comparison of some existing measures. European Journal of Psychological Assessment, 8, 47-56.

O'Donnell, J., \& Anderson, D. (1978). Factors influencing choice of major and career capable women. Vocational Guidance Quarterly, 26, 214-221.

O'Leary, V. (1974). Some attitudinal barriers to occupational aspirations in women. Psychological Bulletin, 81, 809-816.

Olson, M. R., \& Osborne, J. W. (1991). Learning to teach: The first year. Teaching and Teacher Education, 7(4), 331-343.

Pascarella, E. T., \& Terenzini, P. T. (2005). How college affects students: A third decade of research. San Francisco: JosseyBass.

Samuel, M., \& Stephens, D. (2000). Critical dialogues with self: Developing teacher identities and roles - A case study of South African student teachers. International Journal of Educational Research, 33, 475-491.

Savickas, M. L. (2004). Um Modelo para Avaliação de Carreira. In L. Leitão (Ed.), Avaliação Psicológica em orientação escolar e profissional (pp. 21-44). Coimbra: Quarteto.

Savickas, M. L. (2005). The theory and practice of career construction. In D. Brown \& R. Lent (Eds.), Career development and counseling: Putting theory and research to work (pp. 43-70). New Jersey: John Wiley \& Sons, Inc.

Seligman, L. (1994). Developmental career counseling and assessment ( $2^{\text {nd }}$ ed.). London: Sage.

Simões, C. (1996). O desenvolvimento do professor e a construção do conhecimento pedagógico. Aveiro: Fundação João Jacinto de Magalhães.
Simões, C. M., Santos, M. L., Gonçalves, J. A., \& Ralha-Simões, M. H. (1997). A construção da identidade do professor numa perspectiva ecológica de desenvolvimento. In M. F. Patrício (Ed.), Formar professores para a Escola Cultural no horizonte dos anos 2000 (pp.245-260). Porto: Porto Editora.

Slade, P. D., \& Sheehan, M. J. (1979). The measurement of conflict in repertory grids. British Journal of Psychology, 70, 519-524.

Silva, A. D. (2008). A Construção de Carreira no Ensino Superior. Tese de Doutorado, Universidade do Minho, Braga.

Silva, A. D., \& Taveira, A. D. (2005). Grelha de Repertório de Carreira $(G R C)$. Instrumento de Avaliação Não-Publicado. Braga: Centro de Investigação em Psicologia, Universidade do Minho.

SPSS developer's guide. (2005). Chicago, IL: SPSS Inc.

Taveira, M. C. (1997). Exploração e desenvolvimento vocacional de jovens: Estudo sobre as relações entre a exploração, a identidade e a indecisão vocacional. Tese de Doutorado, Universidade do Minho, Braga.

Waterman, A. S., \& Waterman, C. K. (1976). Factors related to vocational identity after extensive work experience. Journal of Applied Psychology, 61, 336-340.

Winter, D. A. (2003). Repertory grid technique as a psychotherapy research measure. Psychotherapy Research, 13, 25-42.

Wortley, D. B., \& Amatea, E. S. (1982). Mapping adult life changes: a conceptual framework for organizing adult development theory. Personnel Guidance Journal, 60, 476-482.

Recebido em 16.01.2009

Primeira decisão editorial em 19.07.2010

Versão final em 29.12.2010

Aceito em 06.04.2011 